\title{
Neutron crystallographic and scattering studies of function and inhibition of HIV-1 protease
}

\author{
Andrey Kovalevsky, ${ }^{1}$ Oksana Gerlits, ${ }^{2}$ Amit Das, ${ }^{3}$ Troy Wymore, ${ }^{4}$ David A. Keen, ${ }^{5}$ Matthew P. \\ Blakeley, ${ }^{6}$ John M. Louis, ${ }^{7}$ Irene Weber, ${ }^{8}$ \\ ${ }^{1}$ Biology and Soft Matter Division, Oak Ridge National Laboratory, Oak Ridge, TN, USA \\ ${ }^{2}$ UT/ORNL Joint Institute for Biological Sciences, University of Tennessee, Knoxville, TN, USA \\ 3Department of Chemistry, University of Michigan, Ann Arbor, MI, USA \\ ${ }^{4}$ Solid State Physics Division, BARC, Trombay, Mumbay, India \\ ${ }^{5}$ ISIS Facility, Rutherford Appleton Laboratory, Harwell Oxford, Didcot, UK \\ ${ }^{6}$ Large-Scale Structures Group, Institut Laue Langevin, Grenoble, France \\ ${ }^{7}$ Laboratory of Chemical Physics, National Institute of Diabetes and Digestive and Kidney Diseases, National Institutes of \\ Health, DHHS, Bethesda, MD, USA \\ ${ }^{8}$ Departments of Chemistry and Biology, Georgia State University, Atlanta, GA, USA
}

There is no cure for HIV-1/AIDS, and a viable vaccine is still years away. Thus, presently, the combination antiretroviral therapy involving treatment with several potent drugs administered simultaneously is the only option available to those infected with HIV-1. Developed drugs target virtually every step in the HIV-1 replication cycle. Such therapeutic intervention has met with significant success, improving the chances of survival and the quality of life for many infected individuals. However, the high mutation rate of HIV-1 coupled with the drug pressure often leads to the development of drug resistant viral strains and, ultimately, therapy failure. HIV-1 drug resistance has become the most significant hurdle to the long-term success of the therapy, demanding development of new anti-HIV-1 drugs. HIV-1 protease enzyme plays a vital role in the viral replication cycle, and has proven to be an effective target for drug design and development, with 9 clinical protease inhibitors currently marketed in the U.S. However, the long-term potency of protease inhibitors is thwarted by rapid emergence of drug resistant protease variants, which necessitates the constant development of new drugs active against resistant protease variants. We are studying HIV-1 protease structure, dynamics, function and the effects of drug resistant mutations on these properties using innovative biophysical methods, including macromolecular neutron crystallography and neutron scattering, in combination with theoretical calculations. We have obtained several room-temperature neutron structures of the wild-type and drug resistant variant HIV-1 protease in complex with clinical inhibitors. Neutron structures directly visualize hydrogen atom positions in the enzyme, revealing fewer hydrogen bonding interactions than was inferred from $100 \mathrm{~K}$ X-ray structures, and demonstrate significant differences between contacts observed at low and room temperatures. Neutrons therefore represent a superb probe to obtain structural details for intermolecular noncovalent interactions and proton transfer reactions in biological systems at a truly atomic level. 